\title{
Acute Effect of Particulate Matter Pollution on Hospital Admissions for Stroke among Patients with type 2 Diabetes in Beijing, China, from 2014 to 2018
}

\section{Xiangtong Liu}

Capital Medical University

\section{Zhiwei Li}

Capital Medical University

\section{Moning Guo}

Beijing Municipal health Commission information center

Jie Zhang

capital medical university

\section{Lixin Tao}

capital medical university

\section{Xiaolin Xu}

the university of queensland

\section{Aklilu Deginet}

Capital Medical University

\section{Feng Lu}

Beijing Municipal Hygiene Bureau Information Center: Beijing Public Health Information Center

\section{Yanxia Luo}

Capital Medical University

\section{Mengmeng Liu}

Capital Medical University

Mengyang Liu

Capital Medical University

\section{Yue Sun}

Capital Medical University

\section{Haibin Li}

Capital Medical University

Xiuhua Guo ( $\nabla$ statguo@ccmu.edu.cn )

School of Public Health, Capital Medical University https://orcid.org/0000-0001-6657-6940 
Research

Keywords: Particulate matter, Stroke, Type 2 diabetes, Comorbidities

Posted Date: January 5th, 2021

DOI: https://doi.org/10.21203/rs.3.rs-137286/v1

License: (c) (i) This work is licensed under a Creative Commons Attribution 4.0 International License. Read Full License 


\section{Abstract}

Background: The health effect of particulate matter pollution on stroke has been widely examined; however, the effect among patients with comorbid type 2 diabetes (T2D) in developing countries has remained largely unknown.

Methods: A time-series study was conducted to investigate the short-term effect of fine particulate matter $\left(\mathrm{PM}_{2.5}\right)$ and inhalable particulate matter $\left(\mathrm{PM}_{10}\right)$ on hospital admissions for stroke among patients with T2D in Beijing, China, from 2014 to 2018. An over-dispersed Poisson generalized additive model was employed to adjust for important covariates, such as weather conditions and long-term and seasonal trends.

Results: A total of 159,298 (58\% male) hospital admissions for stroke were reported. Linear exposureresponse curves were observed for $\mathrm{PM}_{2.5}$ and $\mathrm{PM}_{10}$ in relation to stroke admissions among T2D patients. A $10 \mu \mathrm{g} / \mathrm{m}^{3}$ increase in the four-day moving average of $\mathrm{PM}_{2.5}$ and $\mathrm{PM}_{10}$ was associated with $0.14 \%(95 \%$ confidence interval [Cl]: 0.05\%-0.23\%) and 0.14\% (95\% Cl: 0.06\%-0.22\%) incremental increases in stroke admissions among T2D patients, respectively. A $10 \mu \mathrm{g} / \mathrm{m}^{3}$ increase in $\mathrm{PM}_{2.5}$ in the two-day moving average corresponded to a $0.72 \%$ (95\% Cl: $0.02 \%-1.42 \%)$ incremental increase in hemorrhagic stroke, and a $10 \mu \mathrm{g} / \mathrm{m}^{3}$ increase in $\mathrm{PM}_{10}$ in the four-day moving average corresponded to a $0.14 \%(95 \% \mathrm{Cl}$ : $0.06 \%-0.22 \%$ ) incremental increase in ischemic stroke.

Conclusions: High particulate matter might be a risk factor for stroke among patients with T2D. $\mathrm{PM}_{2.5}$ and $\mathrm{PM}_{10}$ have a linear exposure-response relationship with stroke among T2D patients. The study provided evidence of the risk of comorbid T2D and stroke due to particulate matter pollution.

\section{Highlights}

- The health effect of particulate matter pollution on stroke among the patients with comorbid type 2 diabetes (T2D) in developing countries has remained largely unknown.

- Exposure to particulate matter is associated an increased risk of stroke admissions among the patient with comorbid T2D.

- Linear exposure-response curves were observed between $\mathrm{PM}_{5}$ and $\mathrm{PM}_{10}$ with stroke admissions among T2D patients.

- The study provided evidence of the risk of comorbid T2D and stroke due to particulate matter pollution.

\section{Introduction}

Diabetes mellitus is the ninth major cause of death, and just under half a billion people worldwide were living with diabetes mellitus in 2019, 90\% of whom had type 2 diabetes (T2D) (Saeedi et al., 2019a). Most 
patients with T2D have at least one complication, and stroke is the leading cause of mortality among these patients. China bears the largest stroke burden in the world, which has increased over the past 3 decades (Wang et al., 2017b), accounting for the leading cause of death and disability-adjusted life-years (Zhou et al., 2019). Over one-third of stroke patients have comorbid diabetes, leading to a poor prognosis (Lau et al., 2019). The morbidity of stroke was associated with the progression to diabetes (Xu et al., 2018). To reduce the prevalence of comorbid stroke and diabetes, it is vital to examine modifiable risk factors to formulate appropriate measures for prevention and control.

Air pollutants are common modifiable risk factors for both stroke and T2D (Huang et al., 2019; Paul et al., 2020). Several studies have indicated that ambient air pollution is an important determinant of the risk of stroke (Fisher et al., 2019). Recently, the effect of air pollution on diabetes has attracted increasing attention (Liang et al., 2019; Yang et al., 2018b). Globally, 3.2 million incident cases of diabetes were due to ambient fine particulate matter, leading to 8.2 million disability adjusted life years (Bowe et al., 2018). However, the adverse effects of air pollution on the morbidity of stroke among people with comorbidities in the real world have remained largely unknown. Recently, Xue and colleagues conducted a casecrossover analysis of the China National Stroke Screening Survey and found that the first-ever stroke susceptibility associated with air pollution was significantly varied among Chinese adults (Xue et al., 2019). Li, et al. (Li et al., 2019) reported that people with cardiometabolic comorbidities, especially diabetes, were more vulnerable to air pollution. Epidemiological studies suggest that participants with T2D have lower vascular reactivity and thus may be more susceptible to cardiovascular effects related to air pollution (O'Donnell et al., 2011; O'Neill et al., 2005; Villeneuve et al., 2012).

However, evidence of the association between air pollution and of the risk of stroke among T2D patients is still lacking in China. The burden of comorbidities has become a serious public health problem in many large cities, especially Beijing. However, the acute effects of ambient particulate matter exposure on stroke subtype admissions among T2D participants remain unknown in China.

In this study, we aimed to examine the associations between ambient particulate matter (fine particulate matter with an aerodynamic diameter $<2.5 \mu \mathrm{m}\left[\mathrm{PM}_{2.5}\right]$ and inhalable particulate matter with an aerodynamic diameter $<10 \mu \mathrm{m}\left[\mathrm{PM}_{10}\right]$ ) and total and subtype stroke admissions among patients with T2D in Beijing. In addition, differences in sex and age groups were tested by stratified analyses.

\section{Materials And Methods}

Beijing, located in northern China, had a population of approximately 21.54 million in 2019. The total area of Beijing is 16, 410 square kilometers. Beijing has a rather dry, continental monsoon climate, with four distinct seasons including windy, cold winters and rainy, hot summers.

\subsection{Hospital admission data}


Stoke admission records of patients with T2D were collected from the Beijing Municipal Health Commission Information Center (BMHCIC) (http://www.phic.org.cn/) between Jan 1st, 2014, and Dec 31st, 2018. As a government agency, BMHCIC administers governmental hospitals, covering approximately $95 \%$ of medical services for permanent residents; it should be highly representative of Beijing permanent residents. The geographic locations of the 258 hospitals included in this study have been described elsewhere (Li et al., 2018). The data recording system has shown high validity in our previous study (Aklilu et al., 2020).

Patient data captured from the medical record system included date of admission, T2D status, sex, age, principal diagnosis and secondary diagnosis on discharge. A history of T2D was coded as E12 based on the secondary diagnosis according to the International Classification of Diseases 10th Revision (ICD-10) codes and was defined as having fasting plasma glucose $\geq 7.1 \mathrm{mmol} / \mathrm{L}$ and/or current treatment of diabetes with antidiabetic medication before admission. The subtypes of the stroke hospitalizations were classified as hemorrhagic stroke (ICD-10: 160-162, HS) and ischemic stroke (ICD-10: 163, IS). In the present study, total stroke admissions were calculated as the sum of HS and IS. Only stroke admissions among residents living in Beijing were included in the analysis (Figure S1).

\subsection{Air pollutants and meteorological conditions data}

Daily concentrations of air pollutants including $\mathrm{PM}_{2.5}, \mathrm{PM}_{10}$, nitrogen dioxide $\left(\mathrm{NO}_{2}\right)$, sulfur dioxide $\left(\mathrm{SO}_{2}\right)$, ozone $\left(\mathrm{O}_{3}\right)$, and carbon monoxide (CO) in Beijing were retrieved from the Beijing Environmental Protection Bureau (http://www.bjepb.gov.cn/)

from Jan 1st, 2014, to Dec 31st, 2018. The average concentration of air pollutant data collected was based on 35 ambient air quality monitoring stations. These stations are distributed in 16 districts of Beijing city. The geographic locations of the 35 monitoring stations included in this study have been described elsewhere and have been proven to have good reliability and validity (Huang et al., 2015).

Values for the daily mean temperature and relative humidity data used in the model to adjust for confounders were obtained from the China Meteorological Data Sharing Service System online (https://data.cma.cn/en) over the same time period.

\subsection{Ethical clearance}

The study was approved by the Institutional Review Board of Capital Medical University (No. IRB00009511). Informed consent was not specifically required since personal identifiers were not collected.

\subsection{Statistical analysis}

As the daily counts of hospital admissions generally followed a Poisson distribution (Yu et al., 2019), we applied an over-dispersed generalized additive model (GAM) to examine the short-term effect of particulate matter on stroke admissions among people with T2D (Song et al., 2018). Specifically, covariates were included in the main model as follows: (1) natural smooth functions of temperature and 
relative humidity with 3 degrees of freedom $(d f)$ to control for the nonlinear confounding effects of weather factors (Wang et al., 2017a); (2) indicator variables for public holidays and day of week; and (3) a natural cubic spline function with $7 d f$ for calendar time to exclude unmeasured long-term and seasonal trends of daily stroke admissions (Chen et al., 2018b).

The exposure-response curves were plotted between particulate matter variables and daily stroke admissions of patients with T2D. According to a previous study (Chen et al., 2018a), a natural spline function of $3 d f$ was added into the model. According to the Akaike information criterion, generalized cross validation, and partial autocorrelation function, multiple lag structures including single-day lags (from 0 to 7) and moving average exposure of multiple days (lag $0-1,0-2,0-3,0-4,0-5,0-6,0-7$ ) were used to determine the best lag structure for estimation.

Sensitivity analyses were conducted to examine the robustness of the effect. First, we set the $d f$ value from 4 to 10 per year. Second, principal component analysis was applied to cope with collinearity among air pollutants to estimate the independent effects of particulate matter (Feng et al., 2019) (Stafoggia et al., 2017). For instance, to estimate the independent effects of $\mathrm{PM}_{2.5}$, we first substituted all primary air pollutants $\left(\mathrm{PM}_{10}, \mathrm{NO}_{2}, \mathrm{SO}_{2}, \mathrm{O}_{3}\right.$, and $\left.\mathrm{CO}\right)$ with a composite latent variable (a linear combination of all air pollutants) through principal component analysis, and then included the latent variable in the conditional GAM model. Then the coefficient $\beta^{\prime}$ of the latent variable was transformed into the coefficient $\beta$ of the original air pollutants.

The data were also classified according to sex, age, subtype and T2D comorbid conditions. Patients were classified into two age groups: younger (18-64 years) and elderly ( $\geq 65$ years). $Z$ tests were used to compare differences between effect estimates in the strata (Lin et al., 2016).

The statistical tests were two-sided, and $P<0.05$ was considered statistically significant. All statistical analyses were conducted in $\mathrm{R}$ software (version 4.0.2) using the MGCV package. The effects are described as the percent changes and $95 \% \mathrm{Cl}$ in daily count on admissions for stroke per $10 \mu \mathrm{g} / \mathrm{m}^{3}$ increase in $\mathrm{PM}_{2.5}$ and $\mathrm{PM}_{10}$ among patients with $\mathrm{T} 2 \mathrm{D}$.

\section{Results}

A total of 159,298 admissions for stroke were extracted from BMHCIC between 2014 and 2018; after excluding nonlocal admissions, 149,757 (94\%) hospitalizations for ischemic stroke were included. Male and elderly ( $\geq 65$ years) participants accounted for $58 \%$ and $61 \%$, respectively. Table 1 summarizes the characteristics and distribution of daily stroke admissions, stratified by subtype, sex, and age.

The distribution of air pollutants, meteorological factors, and the counts of daily admissions for stroke across four seasons from 2014 to 2018 are described in Fig. 1. The annual average values of daily mean concentrations of $\mathrm{PM}_{2.5}$ and $\mathrm{PM}_{10}$ were $72.1 \mu \mathrm{g} / \mathrm{m}^{3}$ and $104.5 \mu \mathrm{g} / \mathrm{m}^{3}$, respectively. The concentrations of $\mathrm{PM}_{2.5}$ and $\mathrm{PM}_{10}$ were 1.5 and 2.1 times the Chinese Ambient Air Quality Standards limit, respectively. The 
median temperature was $14.4^{\circ} \mathrm{C}$, and the average relative humidity was $54.3 \pm 19.2 \%$. Air pollutants except $\mathrm{O}_{3}$ were strongly correlated with each other (coefficients distributed from 0.49 to 0.87 ) and moderately correlated with temperature and relative humidity (coefficients distributed from -0.41 to 0.33 ) (Fig. 2).

Table 1

Distribution characteristics of daily stroke events among T2D patients $(n=159,298)$

\begin{tabular}{|c|c|c|c|c|c|c|c|}
\hline & & \multicolumn{2}{|l|}{ Overall stroke } & \multicolumn{2}{|c|}{ Ischemic stroke } & \multicolumn{2}{|c|}{ Hemorrhagic stroke } \\
\hline & & No. (\%) & $P_{25}-P_{75}$ & No. (\%) & $\mathrm{P}_{25}-\mathrm{P}_{75}$ & No. (\%) & $\mathrm{P}_{25}-\mathrm{P}_{75}$ \\
\hline Total & & $159298(100)$ & $56-110$ & 149757 (94) & $52-104$ & $9541(6)$ & $3-7$ \\
\hline \multirow[t]{2}{*}{ Sex } & Male & $92977(58)$ & $33-65$ & $87285(58)$ & $30-61$ & $5692(60)$ & $2-4$ \\
\hline & Female & $66317(42)$ & $24-46$ & $62469(42)$ & $23-44$ & $3848(40)$ & $1-3$ \\
\hline \multirow[t]{2}{*}{ Age (years) } & $18-64$ & $61672(39)$ & $23-43$ & $57454(38)$ & $21-40$ & $4218(44)$ & $1-3$ \\
\hline & $\geq 65$ & $97626(61)$ & $34-68$ & $92303(62)$ & $31-65$ & $5323(56)$ & $2-4$ \\
\hline
\end{tabular}

Table 2 and Table 3 show the effect estimates of $\mathrm{PM}_{2.5}$ and $\mathrm{PM}_{10}$ concentrations on daily stroke admissions among T2D patients according to subtype, sex, and age. Overall, $\mathrm{PM}_{2.5}$ and $\mathrm{PM}_{10}$ were significantly associated with hospitalizations for stroke among T2D patients. According to the smallest parameter value, lags of 4 days (lag4) were determined to be the best lag structure for both $\mathrm{PM}_{2.5}$ and $\mathrm{PM}_{10}$. A $10 \mu \mathrm{g} / \mathrm{m}^{3}$ increase in $\mathrm{PM}_{2.5}$ (lag4) and $\mathrm{PM}_{10}$ (lag4) corresponded to $0.14 \%$ (95\% Cl: $0.05 \%-0.23 \%$ ) and $0.14 \%$ (95\% Cl: $0.06 \%-0.22 \%)$ incremental increased in stroke admissions among diabetic patients, respectively. 
Table 2

Percentage changes $(95 \% \mathrm{Cl})$ in stroke admissions per $10 \mathrm{ug} / \mathrm{m}^{3}$ increase in $\mathrm{PM}_{2.5}$ among T2D patients grouping by sex, age, and subtype.

\begin{tabular}{|c|c|c|c|c|c|c|c|}
\hline $\begin{array}{l}\text { Lag } \\
\text { days }\end{array}$ & Total & $\begin{array}{l}\text { Ischemic } \\
\text { stroke }\end{array}$ & $\begin{array}{l}\text { Hemorrhagic } \\
\text { stroke }\end{array}$ & Men & Women & $\begin{array}{l}\text { Age 18- } \\
64 \mathrm{y}\end{array}$ & $\begin{array}{l}\text { Age } \geq 65 \\
y\end{array}$ \\
\hline $\begin{array}{l}\text { Lag } 0 \\
\text { days }\end{array}$ & $\begin{array}{l}0.18 \\
(-0.02 \\
0.38)\end{array}$ & $\begin{array}{l}0.28 \\
(-0.90 \\
1.48)\end{array}$ & $\begin{array}{l}0.47(-0.36 \\
1.31)\end{array}$ & $\begin{array}{l}0.18 \\
(-0.08, \\
0.45)\end{array}$ & $\begin{array}{l}0.18 \\
(-0.14 \\
0.49)\end{array}$ & $\begin{array}{l}0.39 \\
(0.06 \\
0.71)\end{array}$ & $\begin{array}{l}0.06 \\
(-0.20 \\
0.31)\end{array}$ \\
\hline $\begin{array}{l}\operatorname{Lag} 1 \\
\text { days }\end{array}$ & $\begin{array}{l}0.01 \\
(-0.10 \\
0.11)\end{array}$ & $\begin{array}{l}-0.19 \\
(-0.55 \\
0.17)\end{array}$ & $\begin{array}{l}-0.32(-0.74 \\
0.11)\end{array}$ & $\begin{array}{l}0.04 \\
(-0.10 \\
0.17)\end{array}$ & $\begin{array}{l}-0.03 \\
(-0.20 \\
0.13)\end{array}$ & $\begin{array}{l}0.10 \\
(-0.07 \\
0.27)\end{array}$ & $\begin{array}{l}-0.05 \\
(-0.19 \\
0.08)\end{array}$ \\
\hline $\begin{array}{l}\text { Lag } 2 \\
\text { days }\end{array}$ & $\begin{array}{l}0.01 \\
(-0.08 \\
0.10)\end{array}$ & $\begin{array}{l}-0.09 \\
(-0.40 \\
0.22)\end{array}$ & $\begin{array}{l}-0.14(-0.51 \\
0.22)\end{array}$ & $\begin{array}{l}-0.03 \\
(-0.15 \\
0.09)\end{array}$ & $\begin{array}{l}0.06 \\
(-0.08 \\
0.20)\end{array}$ & $\begin{array}{l}0.06 \\
(-0.08 \\
0.21)\end{array}$ & $\begin{array}{l}-0.02 \\
(-0.14 \\
0.09)\end{array}$ \\
\hline $\begin{array}{l}\text { Lag } 3 \\
\text { days }\end{array}$ & $\begin{array}{l}0.04 \\
(-0.05 \\
0.13)\end{array}$ & $\begin{array}{l}-0.28 \\
(-0.59 \\
0.04)\end{array}$ & $\begin{array}{l}-0.35(-0.72 \\
0.01)\end{array}$ & $\begin{array}{l}0.01 \\
(-0.11 \\
0.13)\end{array}$ & $\begin{array}{l}0.08 \\
(-0.06 \\
0.22)\end{array}$ & $\begin{array}{l}0.12 \\
(-0.02, \\
0.27)\end{array}$ & $\begin{array}{l}-0.01 \\
(-0.13 \\
0.11)\end{array}$ \\
\hline $\begin{array}{l}\text { Lag } 4 \\
\text { days }\end{array}$ & $\begin{array}{l}0.14 \\
(0.05 \\
0.23)\end{array}$ & $\begin{array}{l}0.12 \\
(-0.19 \\
0.43)\end{array}$ & $\begin{array}{l}0.10(-0.26 \\
0.46)\end{array}$ & $\begin{array}{l}0.15 \\
(0.03 \\
0.27)\end{array}$ & $\begin{array}{l}0.11 \\
(-0.03 \\
0.25)\end{array}$ & $\begin{array}{l}0.21 \\
(0.06 \\
0.35)\end{array}$ & $\begin{array}{l}0.09 \\
(-0.03 \\
0.21)\end{array}$ \\
\hline $\begin{array}{l}\text { Lag } 5 \\
\text { days }\end{array}$ & $\begin{array}{l}0.10 \\
(0.01 \\
0.19)\end{array}$ & $\begin{array}{l}0.26 \\
(-0.04, \\
0.56)\end{array}$ & $\begin{array}{l}0.24(-0.11 \\
0.59)\end{array}$ & $\begin{array}{l}0.09 \\
(-0.03 \\
0.20)\end{array}$ & $\begin{array}{l}0.12 \\
(-0.02 \\
0.25)\end{array}$ & $\begin{array}{l}0.12 \\
(-0.02 \\
0.26)\end{array}$ & $\begin{array}{l}0.09 \\
(-0.03 \\
0.20)\end{array}$ \\
\hline $\begin{array}{l}\text { Lag } 6 \\
\text { days }\end{array}$ & $\begin{array}{l}0.11 \\
(0.02 \\
0.19)\end{array}$ & $\begin{array}{l}0.05 \\
(-0.25 \\
0.35)\end{array}$ & $\begin{array}{l}0.16(-0.19, \\
0.51)\end{array}$ & $\begin{array}{l}0.09 \\
(-0.02 \\
0.21)\end{array}$ & $\begin{array}{l}0.13 \\
(-0.01 \\
0.26)\end{array}$ & $\begin{array}{l}0.14 \\
(0.00 \\
0.28)\end{array}$ & $\begin{array}{l}0.08 \\
(-0.03 \\
0.20)\end{array}$ \\
\hline $\begin{array}{l}\text { Lag } 7 \\
\text { days }\end{array}$ & $\begin{array}{l}0.07 \\
(-0.02 \\
0.16)\end{array}$ & $\begin{array}{l}0.19 \\
(-0.11 \\
0.49)\end{array}$ & $\begin{array}{l}0.26(-0.09 \\
0.62)\end{array}$ & $\begin{array}{l}0.07 \\
(-0.04 \\
0.19)\end{array}$ & $\begin{array}{l}0.06 \\
(-0.07 \\
0.20)\end{array}$ & $\begin{array}{l}0.11 \\
(-0.03 \\
0.25)\end{array}$ & $\begin{array}{l}0.04 \\
(-0.07 \\
0.16)\end{array}$ \\
\hline $\begin{array}{l}\text { Lag } 0- \\
1 \text { days }\end{array}$ & $\begin{array}{l}0.10 \\
(-0.07 \\
0.27)\end{array}$ & $\begin{array}{l}0.62 \\
(-0.04, \\
1.29)\end{array}$ & $\begin{array}{l}0.72(0.02, \\
1.42)\end{array}$ & $\begin{array}{l}0.12 \\
(-0.11, \\
0.34)\end{array}$ & $\begin{array}{l}0.08 \\
(-0.18 \\
0.35)\end{array}$ & $\begin{array}{l}0.09 \\
(-0.18 \\
0.37)\end{array}$ & $\begin{array}{l}0.11 \\
(-0.11 \\
0.33)\end{array}$ \\
\hline $\begin{array}{l}\text { Lag } 0- \\
2 \text { days }\end{array}$ & $\begin{array}{l}0.09 \\
(-0.10 \\
0.29)\end{array}$ & $\begin{array}{l}0.28 \\
(-0.48 \\
1.04)\end{array}$ & $\begin{array}{l}0.27(-0.51 \\
1.07)\end{array}$ & $\begin{array}{l}0.13 \\
(-0.13 \\
0.38)\end{array}$ & $\begin{array}{l}0.04 \\
(-0.26 \\
0.34)\end{array}$ & $\begin{array}{l}0.18 \\
(-0.13 \\
0.49)\end{array}$ & $\begin{array}{l}0.04 \\
(-0.21 \\
0.29)\end{array}$ \\
\hline $\begin{array}{l}\text { Lag } 0- \\
3 \text { days }\end{array}$ & $\begin{array}{l}0.02 \\
(-0.16 \\
0.21)\end{array}$ & $\begin{array}{l}0.31 \\
(-0.37 \\
0.99)\end{array}$ & $\begin{array}{l}0.46(-0.29 \\
1.22)\end{array}$ & $\begin{array}{l}0.06 \\
(-0.19 \\
0.30)\end{array}$ & $\begin{array}{l}-0.02 \\
(-0.31 \\
0.26)\end{array}$ & $\begin{array}{l}0.10 \\
(-0.19 \\
0.40)\end{array}$ & $\begin{array}{l}-0.03 \\
(-0.26 \\
0.21)\end{array}$ \\
\hline $\begin{array}{l}\text { Lag } 0- \\
4 \text { days }\end{array}$ & $\begin{array}{l}0.06 \\
(-0.13 \\
0.26)\end{array}$ & $\begin{array}{l}0.26 \\
(-0.43 \\
0.95)\end{array}$ & $\begin{array}{l}0.35(-0.43 \\
1.14)\end{array}$ & $\begin{array}{l}0.07 \\
(-0.19 \\
0.32)\end{array}$ & $\begin{array}{l}0.06 \\
(-0.24 \\
0.36)\end{array}$ & $\begin{array}{l}0.18 \\
(-0.14 \\
0.49)\end{array}$ & $\begin{array}{l}0.00 \\
(-0.25 \\
0.24)\end{array}$ \\
\hline
\end{tabular}

* Statistically positive significant results at the $5 \%$ level $(P<0.05)$ are indicated in bold. T2D: type 2 diabetes. 


\begin{tabular}{|c|c|c|c|c|c|c|c|}
\hline $\begin{array}{l}\text { Lag } \\
\text { days }\end{array}$ & Total & $\begin{array}{l}\text { Ischemic } \\
\text { stroke }\end{array}$ & $\begin{array}{l}\text { Hemorrhagic } \\
\text { stroke }\end{array}$ & Men & Women & $\begin{array}{l}\text { Age 18- } \\
64 \mathrm{y}\end{array}$ & $\begin{array}{l}\text { Age } \geq 65 \\
y\end{array}$ \\
\hline $\begin{array}{l}\text { Lag 0- } \\
5 \text { days }\end{array}$ & $\begin{array}{l}-0.02 \\
(-0.22 \\
0.18)\end{array}$ & $\begin{array}{l}0.51 \\
(-0.18 \\
1.21)\end{array}$ & $\begin{array}{l}0.51(-0.29 \\
1.32)\end{array}$ & $\begin{array}{l}-0.07 \\
(-0.33 \\
0.19)\end{array}$ & $\begin{array}{l}0.05 \\
(-0.25 \\
0.36)\end{array}$ & $\begin{array}{l}0.10 \\
(-0.22, \\
0.42)\end{array}$ & $\begin{array}{l}-0.09 \\
(-0.34 \\
0.17)\end{array}$ \\
\hline $\begin{array}{l}\text { Lag } 0- \\
6 \text { days }\end{array}$ & $\begin{array}{l}0.03 \\
(-0.18 \\
0.24)\end{array}$ & $\begin{array}{l}0.34 \\
(-0.38 \\
1.07)\end{array}$ & $\begin{array}{l}0.28(-0.56 \\
1.13)\end{array}$ & $\begin{array}{l}-0.05 \\
(-0.32 \\
0.23)\end{array}$ & $\begin{array}{l}0.14 \\
(-0.18 \\
0.46)\end{array}$ & $\begin{array}{l}0.21 \\
(-0.13 \\
0.54)\end{array}$ & $\begin{array}{l}-0.08 \\
(-0.35 \\
0.19)\end{array}$ \\
\hline $\begin{array}{l}\text { Lag 0- } \\
7 \text { days }\end{array}$ & $\begin{array}{l}-0.03 \\
(-0.25 \\
0.19)\end{array}$ & $\begin{array}{l}0.43 \\
(-0.33 \\
1.19)\end{array}$ & $\begin{array}{l}0.28(-0.60 \\
1.17)\end{array}$ & $\begin{array}{l}-0.11 \\
(-0.39 \\
0.18)\end{array}$ & $\begin{array}{l}0.07 \\
(-0.27 \\
0.41)\end{array}$ & $\begin{array}{l}0.06 \\
(-0.30 \\
0.41)\end{array}$ & $\begin{array}{l}-0.09 \\
(-0.37 \\
0.19)\end{array}$ \\
\hline
\end{tabular}


Table 3

Percentage changes $(95 \% \mathrm{Cl})$ in stroke admissions per $10 \mathrm{ug} / \mathrm{m}^{3}$ increase in $\mathrm{PM}_{10}$ among T2D patients grouping by sex, age, and subtype.

\begin{tabular}{|c|c|c|c|c|c|c|c|}
\hline $\begin{array}{l}\text { Lag } \\
\text { days }\end{array}$ & Total & $\begin{array}{l}\text { Ischemic } \\
\text { stroke }\end{array}$ & $\begin{array}{l}\text { Hemorrhagic } \\
\text { stroke }\end{array}$ & Men & Women & $\begin{array}{l}\text { Age 18- } \\
64 \mathrm{y}\end{array}$ & $\begin{array}{l}\text { Age } \geq 65 \\
y\end{array}$ \\
\hline $\begin{array}{l}\text { Lag } 0 \\
\text { days }\end{array}$ & $\begin{array}{l}0.17 \\
(-0.12 \\
0.46)\end{array}$ & $\begin{array}{l}0.16 \\
(-0.13 \\
0.46)\end{array}$ & $\begin{array}{l}0.28(-0.90 \\
1.48)\end{array}$ & $\begin{array}{l}0.25 \\
(-0.12 \\
0.63)\end{array}$ & $\begin{array}{l}0.06 \\
(-0.39 \\
0.50)\end{array}$ & $\begin{array}{l}0.23 \\
(-0.24 \\
0.69)\end{array}$ & $\begin{array}{l}0.14 \\
(-0.23 \\
0.50)\end{array}$ \\
\hline $\begin{array}{l}\text { Lag } 1 \\
\text { days }\end{array}$ & $\begin{array}{l}0.03 \\
(-0.05 \\
0.12)\end{array}$ & $\begin{array}{l}0.05 \\
(-0.04, \\
0.14)\end{array}$ & $\begin{array}{l}-0.19(-0.55 \\
0.17)\end{array}$ & $\begin{array}{l}0.05 \\
(-0.07 \\
0.16)\end{array}$ & $\begin{array}{l}0.02 \\
(-0.12 \\
0.15)\end{array}$ & $\begin{array}{l}0.13 \\
(-0.01 \\
0.27)\end{array}$ & $\begin{array}{l}-0.02 \\
(-0.14 \\
0.09)\end{array}$ \\
\hline $\begin{array}{l}\text { Lag } 2 \\
\text { days }\end{array}$ & $\begin{array}{l}0.02 \\
(-0.06 \\
0.09)\end{array}$ & $\begin{array}{l}0.02 \\
(-0.05 \\
0.10)\end{array}$ & $\begin{array}{l}-0.09 \\
0.22)\end{array}$ & $\begin{array}{l}-0.01 \\
(-0.11 \\
0.09)\end{array}$ & $\begin{array}{l}0.06 \\
(-0.06 \\
0.18)\end{array}$ & $\begin{array}{l}0.07 \\
(-0.05 \\
0.20)\end{array}$ & $\begin{array}{l}-0.02 \\
(-0.12 \\
0.08)\end{array}$ \\
\hline $\begin{array}{l}\text { Lag } 3 \\
\text { days }\end{array}$ & $\begin{array}{l}0.06 \\
(-0.02 \\
0.14)\end{array}$ & $\begin{array}{l}0.08 \\
(0.00 \\
0.17)\end{array}$ & $\begin{array}{l}-0.28(-0.59 \\
0.04)\end{array}$ & $\begin{array}{l}0.04 \\
(-0.06 \\
0.14)\end{array}$ & $\begin{array}{l}0.09 \\
(-0.03 \\
0.21)\end{array}$ & $\begin{array}{l}0.15 \\
(0.03, \\
0.28)\end{array}$ & $\begin{array}{l}0.00 \\
(-0.10 \\
0.10)\end{array}$ \\
\hline $\begin{array}{l}\text { Lag } 4 \\
\text { days }\end{array}$ & $\begin{array}{l}0.14 \\
(0.06 \\
0.22)\end{array}$ & $\begin{array}{l}0.14 \\
(0.06 \\
0.22)\end{array}$ & $\begin{array}{l}0.12(-0.19, \\
0.43)\end{array}$ & $\begin{array}{l}0.14 \\
(0.04 \\
0.24)\end{array}$ & $\begin{array}{l}0.14 \\
(0.02, \\
0.26)\end{array}$ & $\begin{array}{l}0.20 \\
(0.08 \\
0.33)\end{array}$ & $\begin{array}{l}0.10 \\
(0.00 \\
0.20)\end{array}$ \\
\hline $\begin{array}{l}\text { Lag } 5 \\
\text { days }\end{array}$ & $\begin{array}{l}0.12 \\
(0.04 \\
0.19)\end{array}$ & $\begin{array}{l}0.11 \\
(0.03 \\
0.18)\end{array}$ & $\begin{array}{l}0.26(-0.04 \\
0.56)\end{array}$ & $\begin{array}{l}0.09 \\
(-0.01 \\
0.19)\end{array}$ & $\begin{array}{l}0.15 \\
(0.04 \\
0.27)\end{array}$ & $\begin{array}{l}0.13 \\
(0.01, \\
0.25)^{\prime}\end{array}$ & $\begin{array}{l}0.11 \\
(0.01, \\
0.20)^{\prime}\end{array}$ \\
\hline $\begin{array}{l}\text { Lag } 6 \\
\text { days }\end{array}$ & $\begin{array}{l}0.11 \\
(0.04 \\
0.18)\end{array}$ & $\begin{array}{l}0.11 \\
(0.04, \\
0.19)\end{array}$ & $\begin{array}{l}0.05(-0.25 \\
0.35)\end{array}$ & $\begin{array}{l}0.08 \\
(-0.01, \\
0.18)\end{array}$ & $\begin{array}{l}0.15 \\
(0.03 \\
0.26)\end{array}$ & $\begin{array}{l}0.10 \\
(-0.02, \\
0.22)\end{array}$ & $\begin{array}{l}0.12 \\
(0.02 \\
0.21)\end{array}$ \\
\hline $\begin{array}{l}\text { Lag } 7 \\
\text { days }\end{array}$ & $\begin{array}{l}0.08 \\
(0.00 \\
0.16)\end{array}$ & $\begin{array}{l}0.07 \\
(-0.01 \\
0.15)\end{array}$ & $\begin{array}{l}0.19(-0.11 \\
0.49)\end{array}$ & $\begin{array}{l}0.10 \\
(0.00 \\
0.20)\end{array}$ & $\begin{array}{l}0.06 \\
(-0.06 \\
0.17)\end{array}$ & $\begin{array}{l}0.08 \\
(-0.04 \\
0.20)\end{array}$ & $\begin{array}{l}0.08 \\
(-0.01 \\
0.18)\end{array}$ \\
\hline $\begin{array}{l}\text { Lag } 0- \\
1 \text { days }\end{array}$ & $\begin{array}{l}0.14 \\
(-0.03 \\
0.30)\end{array}$ & $\begin{array}{l}0.11 \\
(-0.06, \\
0.27)\end{array}$ & $\begin{array}{l}0.62(-0.04 \\
1.29)\end{array}$ & $\begin{array}{l}0.16 \\
(-0.05 \\
0.38)\end{array}$ & $\begin{array}{l}0.10 \\
(-0.16, \\
0.35)\end{array}$ & $\begin{array}{l}0.08 \\
(-0.18 \\
0.35)\end{array}$ & $\begin{array}{l}0.17 \\
(-0.04, \\
0.38)\end{array}$ \\
\hline $\begin{array}{l}\text { Lag } 0- \\
2 \text { days }\end{array}$ & $\begin{array}{l}0.15 \\
(-0.03 \\
0.34)\end{array}$ & $\begin{array}{l}0.15 \\
(-0.05 \\
0.34)\end{array}$ & $\begin{array}{l}0.28(-0.48 \\
1.04)\end{array}$ & $\begin{array}{l}0.18 \\
(-0.07 \\
0.43)\end{array}$ & $\begin{array}{l}0.12 \\
(-0.17 \\
0.41)\end{array}$ & $\begin{array}{l}0.24 \\
(-0.06 \\
0.54)\end{array}$ & $\begin{array}{l}0.10 \\
(-0.14, \\
0.34)\end{array}$ \\
\hline $\begin{array}{l}\text { Lag } 0- \\
3 \text { days }\end{array}$ & $\begin{array}{l}0.10 \\
(-0.07 \\
0.26)\end{array}$ & $\begin{array}{l}0.08 \\
(-0.09 \\
0.26)\end{array}$ & $\begin{array}{l}0.31(-0.37 \\
0.99)\end{array}$ & $\begin{array}{l}0.12 \\
(-0.09, \\
0.34)\end{array}$ & $\begin{array}{l}0.06 \\
(-0.20 \\
0.32)\end{array}$ & $\begin{array}{l}0.19 \\
(-0.08, \\
0.46)\end{array}$ & $\begin{array}{l}0.04 \\
(-0.18 \\
0.25)\end{array}$ \\
\hline $\begin{array}{l}\text { Lag } 0- \\
4 \text { days }\end{array}$ & $\begin{array}{l}0.11 \\
(-0.06 \\
0.28)\end{array}$ & $\begin{array}{l}0.10 \\
(-0.08, \\
0.27)\end{array}$ & $\begin{array}{l}0.26(-0.43 \\
0.95)\end{array}$ & $\begin{array}{l}0.09 \\
(-0.13, \\
0.31)\end{array}$ & $\begin{array}{l}0.13 \\
(-0.14, \\
0.39)\end{array}$ & $\begin{array}{l}0.23 \\
(-0.04, \\
0.50)\end{array}$ & $\begin{array}{l}0.03 \\
(-0.19 \\
0.24)\end{array}$ \\
\hline
\end{tabular}

* Statistically positive significant results at the $5 \%$ level $(P<0.05)$ are indicated in bold. 


\begin{tabular}{|c|c|c|c|c|c|c|c|}
\hline $\begin{array}{l}\text { Lag } \\
\text { days }\end{array}$ & Total & $\begin{array}{l}\text { Ischemic } \\
\text { stroke }\end{array}$ & $\begin{array}{l}\text { Hemorrhagic } \\
\text { stroke }\end{array}$ & Men & Women & $\begin{array}{l}\text { Age 18- } \\
64 \mathrm{y}\end{array}$ & $\begin{array}{l}\text { Age } \geq 65 \\
y\end{array}$ \\
\hline $\begin{array}{l}\text { Lag } 0- \\
5 \text { days }\end{array}$ & $\begin{array}{l}0.01 \\
(-0.16 \\
0.18)\end{array}$ & $\begin{array}{l}-0.02 \\
(-0.20 \\
0.15)\end{array}$ & $\begin{array}{l}0.51(-0.18 \\
1.21)\end{array}$ & $\begin{array}{l}-0.05 \\
(-0.28 \\
0.17)\end{array}$ & $\begin{array}{l}0.09 \\
(-0.17 \\
0.36)\end{array}$ & $\begin{array}{l}0.12 \\
(-0.16 \\
0.40)\end{array}$ & $\begin{array}{l}-0.06 \\
(-0.28 \\
0.16)\end{array}$ \\
\hline $\begin{array}{l}\text { Lag } 0- \\
6 \text { days }\end{array}$ & $\begin{array}{l}0.04 \\
(-0.14, \\
0.22)\end{array}$ & $\begin{array}{l}0.02 \\
(-0.16 \\
0.21)\end{array}$ & $\begin{array}{l}0.34(-0.38 \\
1.07)\end{array}$ & $\begin{array}{l}-0.05 \\
(-0.29 \\
0.18)\end{array}$ & $\begin{array}{l}0.17 \\
(-0.10 \\
0.45)\end{array}$ & $\begin{array}{l}0.21 \\
(-0.08 \\
0.50)\end{array}$ & $\begin{array}{l}-0.06 \\
(-0.29 \\
0.17)\end{array}$ \\
\hline $\begin{array}{l}\text { Lag 0- } \\
7 \text { days }\end{array}$ & $\begin{array}{l}-0.08 \\
(-0.27 \\
0.11)\end{array}$ & $\begin{array}{l}-0.11 \\
(-0.30 \\
0.09)\end{array}$ & $\begin{array}{l}0.43(-0.33 \\
1.19)\end{array}$ & $\begin{array}{l}-0.16 \\
(-0.40 \\
0.09)\end{array}$ & $\begin{array}{l}0.04 \\
(-0.26 \\
0.33)\end{array}$ & $\begin{array}{l}0.03 \\
(-0.27 \\
0.34)\end{array}$ & $\begin{array}{l}-0.15 \\
(-0.39 \\
0.10)\end{array}$ \\
\hline
\end{tabular}

Specifically, for $\mathrm{PM}_{2.5}$, a $10 \mu \mathrm{g} / \mathrm{m}^{3}$ increase corresponded to a $0.72 \%$ (95\% Cl: 0.02\%-1.42\%) incremental increase in hemorrhagic stroke (lag $0-1)$. The incremental increase in hemorrhagic stroke was $0.15 \%$ (95\% Cl: $0.03 \%-0.27 \%$ ) in men (lag 4), and $0.39 \%$ (95\% Cl: $0.06 \%-0.71 \%$ ) in participants aged $18-64$ years (lag 0) (Table 2). For $\mathrm{PM}_{10}$, a $10 \mu \mathrm{g} / \mathrm{m}^{3}$ increase in the four-day moving average corresponded to a $0.14 \%$ (95\% Cl: $0.06 \%-0.22 \%)$ incremental increase in ischemic stroke. The effect size was $0.14 \%$ (95\% Cl: $0.04 \%-0.24 \%$ ) for men and 0.15 (95\% Cl: $0.04 \%-0.27 \%)$ for women, and the difference was not statistically significant $(Z=-0.29, P=0.77)$. The effect size in participants aged $18-64$ years was significantly higher than that in participants aged $\geq 65$ years $[0.20 \%$ (95\% Cl: $0.08 \%-0.33 \%)$ vs. $0.12 \%(95 \% \mathrm{Cl}: 0.02 \%-0.21 \%)$, $Z=-1.62, P=0.10$ ] (Table 3). Subgroup analysis indicated that the onset of the maximum effect of $\mathrm{PM}_{10}$ was much earlier for males and younger adults than for females and elderly adults (Table 3 ).

The exposure-response curves of the overall association between $\mathrm{PM}_{2.5}$ and stroke admissions among T2D patients are shown in Fig. 3. Monotonic increasing curves were observed overall and in each of the subgroups of T2D patients, which clearly indicated that the effect of $\mathrm{PM}_{2.5}$ on stroke is linear. Evidently, high risks were observed for high $\mathrm{PM}_{2.5}$ concentrations, with flat slopes at low concentrations and steeper slopes for concentrations $\geq 200 \mu \mathrm{g} / \mathrm{m}^{3}$.

The exposure-response curves for the overall association between $\mathrm{PM}_{10}$ and stroke admissions among T2D patients are shown in Fig. 4. Monotonic increasing curves were observed overall and in subgroups of T2D patients, which indicated that the effect of $\mathrm{PM}_{10}$ on stroke is almost linear. Evidently, high risks were observed for high $\mathrm{PM}_{10}$ concentrations, with flat slopes at low concentrations and steeper slopes for concentrations $\geq 200 \mu \mathrm{g} / \mathrm{m}^{3}$.

\section{Discussion}

To the best of our knowledge, this is the first study that attempted to determine associations between particulate matter pollution and stroke admissions among patients with T2D in China. Generally, this research showed that short-term exposure to particulate matter pollution was associated with an 
increased risk of stroke admissions among patients with T2D. The associations between particulate matter and stroke admissions in patients with T2D varied by sex, age and subtype, and the associations were robust when adjusted for copollutants. This study added to the limited evidence of acute effects of particulate matter pollution in populations with comorbidities in developing countries.

Between 2014 and 2018, the average concentrations of $\mathrm{PM}_{2.5}\left(72.1 \mu \mathrm{g} / \mathrm{m}^{3}\right)$ and $\mathrm{PM}_{10}\left(104.5 \mu \mathrm{g} / \mathrm{m}^{3}\right)$ in Beijing far exceeded the Chinese National Ambient Air Quality Standards $\left(35 \mu \mathrm{g} / \mathrm{m}^{3}\right.$ and $70 \mu \mathrm{g} / \mathrm{m}^{3}$, respectively). Meanwhile, T2D has become the leading risk factor for cerebrovascular disease in the Chinese population. It is estimated that 116.4 million Chinese adults had T2D in 2019, ranking first in the world (Saeedi et al., 2019b). Although the adverse effect attributable to PM exposure might be small in an individual, the overall attributable risk might be considerably higher, given the high pollutant concentrations and large population of T2D in China (Maji et al., 2017; Maji et al., 2018).

The number of studies on the short-term effect of particulate matter on stroke has increased during the past few years. Significant associations were observed between particulate matter $\left(\mathrm{PM}_{2.5}\right.$ and $\left.\mathrm{PM}_{10}\right)$ concentrations and admissions for stroke, which are roughly consistent with results from previous studies (Huang et al., 2016; Tian et al., 2017). Liu et al found that an interquartile range increase in $\mathrm{PM}_{2.5}$ $\left(47.5 \mu \mathrm{g} / \mathrm{m}^{3}\right)$ and $\mathrm{PM}_{10}\left(76.9 \mu \mathrm{g} / \mathrm{m}^{3}\right)$ was significantly associated with admissions for ischemic stroke, with per centage changes of $1.0 \%$ (95\% Cl: $0.7 \%-1.4 \%$ ) and $0.8 \%$ (95\% Cl: $0.3 \%-1.3 \%)$, respectively (Liu et al., 2017b). Previous research showed that short-term exposure to $\mathrm{PM}_{2.5}$ and $\mathrm{PM}_{10}$ was positively related to stroke admission and mortality (RRs: 1.011 (95\% Cl: 1.011-1.012) and 1.003 (95\% Cl: 1.002-1.004), respectively) (Shah et al., 2015). An interquartile range of $\mathrm{PM}_{10}$ corresponded to $0.7 \%$ (95\% Cl: $0 \%-1.4 \%$ ) increases in admissions for ischemic stroke (Liu et al., 2017a).

We found that for patients with comorbid T2D, the effect estimates for both $\mathrm{PM}_{2.5}$ and $\mathrm{PM}_{10}$ using moving average lags were much higher than those using single day lags. Most previous studies were designed to study the effects of particulate matter on the morbidity or mortality of stroke. Our study suggests that for participants with T2D, more attention should be paid to the health hazards of air pollution. Similarly, Yang et al. (Yang et al., 2020) found that patients with T2D might be more vulnerable to air pollutant exposure, and Paul et al. found that exposure to PM was associated with an increased risk of cardiovascular diseases among populations with prevalent T2D (Paul et al., 2020).

Even if the exact mechanisms behind the association of air pollution with increased admission in the T2D group have not been determined, there is growing evidence suggesting that the diabetes effect of PM pollution might be caused by endothelial dysfunction and systemic proinflammatory processes, leading to insulin resistance (Rajagopalan et al., 2018). Alterations in metabolic and inflammatory processes are crucial intermediate steps in explaining how PM exposure increases the risk of T2D, which is characterized by low-grade systemic inflammation (Lao et al., 2019). Previous research suggests that direct exposure of the respiratory tract to PM produces local inflammatory responses in lung tissue (Honda et al., 2017). It is hypothesized that upon chronic PM exposure, these local inflammatory 
processes spread to other organ systems and then lead to blood glucose metabolism abnormalities (Hotamisligil, 2017). A recent study indicated that adiponectin may be a potential mediator along the causal pathway (Lucht et al., 2020). These mechanisms might overlap at different times, and further studies are warranted to clarify them in detail.

The effect size of $\mathrm{PM}_{2.5}$ was much higher than that of $\mathrm{PM}_{10}$, which is generally consistent with existing studies (Huang et al., 2016; Zeng et al., 2018). Several mechanisms were proposed to explain the difference. First, $\mathrm{PM}_{2.5}$ can penetrate the systemic circulation and then reach their way into the alveoli as well, whereas $\mathrm{PM}_{10}$ can enter only the respiratory tract. Second, $\mathrm{PM}_{2.5}$ has a higher particulate number and larger active surface area than $\mathrm{PM}_{10}$. Third, the larger surface area could carry a much larger volume of toxic pollutants to generate proinflammatory responses.

Consistent with a previous study (Zeng et al., 2018), higher effect estimates were reported in younger adults (18-64 years). The reasons for the difference between age groups remain unknown. As air pollution has become an increasingly serious public health problem during the past few decades, and younger generations may suffer from air pollution earlier in their life cycle. Furthermore, older participants may pay more attention to preventing environmental hazards from PM pollution. For example, older participants are prone to stay at home during heavy pollution days.

The present study showed the increased effect of air pollution exposure on stroke admissions among patients with T2D. The inhalation of toxic substances in individuals with compromised metabolic function due to diabetes may contribute to stroke by weakening the immune system and further decreasing metabolic function. In our study, the effect of particulate matter on admissions for overall stroke varied by sex among patients with T2D. Consistent with previous research, we found that $\mathrm{PM}_{2.5}$ had stronger effects in male participants, whereas $\mathrm{PM}_{10}$ had stronger effects in female participants. Hwang and colleagues demonstrated that the sex-specific effects could be explained by biological differences as well as differences in social exposures (Hwang et al., 2020). Generally, lung size, gas-blood barrier permeability and inflammation all vary by sex. Xia reported that, among surviving stroke patients, women were more susceptible to diabetes than men (Xia et al., 2019). Furthermore, men participate in more outdoor activities with fewer personal safeguard measures (e.g. wearing particulate-filtering masks), leading to increased exposure to particulate matter (Yang et al., 2018a).

The determination of exposure-response relationships is critical for public health assessment of those with preexisting noncommunicable diseases. In our study, linear associations were observed between particulate matter and daily stroke admissions among patients with T2D. Consistent with a previous study, monotonically increasing curves were observed overall and in each of the subgroups of T2D patients which clearly suggests that the effect of both $\mathrm{PM}_{2.5}$ and $\mathrm{PM}_{10}$ on stroke is linear (Costa et al., 2017). Particulate matter concentrations should be further limited to protect people with T2D, although exposure-response relationships may differ by air pollution mixture, meteorological conditions, and population susceptibility (Song et al., 2019). 
The study had some limitations. First, we could not collect data on the relationships between patients. However, there might be a few family aggregation cases, which may affect the population sensitivity (Yang et al., 2018c). Second, we used fixed-site monitor measurements as a proxy for personal exposure, which may result in exposure errors and underestimation of the associations between ambient air pollution and diseases. Third, the generalizability of our results might be limited as the study collected data from only one highly polluted city.

\section{Conclusions}

This study suggests that short-term exposure to ambient $\mathrm{PM}_{2.5}$ and $\mathrm{PM}_{10}$ significantly increased the risk of stroke admissions among patients with T2D. Linear exposure-response curves were observed for $\mathrm{PM}_{2.5}$ and $\mathrm{PM}_{10}$ in relation to stroke admissions among T2D patients, with flat slopes at low concentrations and steeper slopes for high concentrations. High particulate matter concentrations might be one risk factor for stroke among patients with T2D in Beijing, China. The study provided evidence of the risk of comorbidities due to particulate matter pollution.

\section{Abbreviations}

T2D: type 2 diabetes; $\mathrm{PM}_{2.5}$ : fine particulate matter with an aerodynamic diameter $<2.5 \mu \mathrm{m} ; \mathrm{PM}_{10}$ : inhalable particulate matter with an aerodynamic diameter $<10 \mu \mathrm{m} ; 95 \% \mathrm{Cl}$ : 95\% confidence interval; BMHCIC: the Beijing Municipal Health Commission Information Center; ICD-10: the International Classification of Diseases, $10^{\text {th }}$ Revision; $\mathrm{HS}$ : hemorrhagic stroke; IS: ischemic stroke; $\mathrm{NO}_{2}$ : nitrogen dioxide; $\mathrm{SO}_{2}$ : sulfur dioxide; $\mathrm{O}_{3}$ : ozone; $\mathrm{CO}$ : carbon monoxide; GAM: generalized additive model; $d f$. degrees of freedom; SD: standard deviation; IQR: interquartile range.

\section{Declarations}

\section{Acknowledgments}

We acknowledge the Beijing Municipal Health Commission Information Center and the Beijing Air Pollution and Metrological Data Center.

\section{Funding}

This study was supported by National Natural Science Foundation of China (No. 82003559), Nature Science Foundation of Capital Medical University (No. PYZ2018046), Beijing Municipal Training Project of Excellent Talents. The funding was neither used for the study design nor data collection but to cover for the publication fees. 


\section{Availability of data and materials}

The data can be accessed from the Beijing Municipal Health Commission Information Center with permission via direct request.

\section{Authors' contributions}

Xiangtong Liu: Conceptualization, Methodology, Data curation, Writing- Original draft preparation, Funding acquisition; Zhiwei Li: Methodology, Software, Data curation, Visualization; Moning Guo: Data curation, Resources; Jie Zhang: Supervision, Data curation; Lixin Tao: Writing- Reviewing and Editing; Xiaolin Xu: Writing- Reviewing and Editing; Aklilu Deginet: Writing- Reviewing and Editing; Feng Lu: Resources; Yanxia Luo: Conceptualization, Methodology; Mengmeng Liu: Formal analysis; Mengyang Liu: Formal analysis; Yue Sun: Formal analysis; Haibin Li: Formal analysis; Xiuhua Guo: Supervision, WritingReviewing and Editing. All authors were involved with the critical revision of the manuscript and approved the final version.

\section{Ethics approval}

In this study, informed consent was not specifically required because we did not use personal data identifiers. However, the study was approved by the Institutional Review Board of Capital Medical University with the IRB00009511 identification number.

\section{Consent for publication}

Not applicable.

\section{Competing interests}

The authors declare that they have no competing interests.

\section{References}

1. Aklilu, D., et al., 2020. Short-term effects of extreme temperatures on cause specific cardiovascular admissions in Beijing, China. Environ Res. 186, 109455.

2. Bowe, B., et al., 2018. The 2016 global and national burden of diabetes mellitus attributable to PM2.5 air pollution. Lancet Planet Health. 2, e301-e312.

3. Chen, C., et al., 2018a. Ambient air pollution and daily hospital admissions for mental disorders in Shanghai, China. Sci Total Environ. 613-614, 324-330. 
4. Chen, F., et al., 2018b. The effects of PM2.5 on asthmatic and allergic diseases or symptoms in preschool children of six Chinese cities, based on China, Children, Homes and Health (CCHH) project. Environ Pollut. 232, 329-337.

5. Costa, A. F., et al., 2017. Air Pollution and Deaths among Elderly Residents of São Paulo, Brazil: An Analysis of Mortality Displacement. Environ Health Perspect. 125, 349-354.

6. Feng, W., et al., 2019. Short-term PM10 and emergency department admissions for selective cardiovascular and respiratory diseases in Beijing, China. Sci Total Environ. 657, 213-221.

7. Fisher, J. A., et al., 2019. Case-crossover analysis of short-term particulate matter exposures and stroke in the health professionals follow-up study. Environ Int. 124, 153-160.

8. Honda, T., et al., 2017. Associations between long-term exposure to air pollution, glycosylated hemoglobin and diabetes. Int J Hyg Environ Health. 220, 1124-1132.

9. Hotamisligil, G. S., 2017. Inflammation, metaflammation and immunometabolic disorders. Nature. $542,177-185$.

10. Huang, F., et al., 2015. PM2.5 Spatiotemporal Variations and the Relationship with Meteorological Factors during 2013-2014 in Beijing, China. PLoS One. 10, e0141642.

11. Huang, F., et al., 2016. Particulate Matter and Hospital Admissions for Stroke in Beijing, China: Modification Effects by Ambient Temperature. J Am Heart Assoc. 5.

12. Huang, K., et al., 2019. Long term exposure to ambient fine particulate matter and incidence of stroke: prospective cohort study from the China-PAR project. BMJ. 367, 16720.

13. Hwang, M. J., et al., 2020. Impacts of ambient air pollution on glucose metabolism in Korean adults: a Korea National Health and Nutrition Examination Survey study. Environ Health. 19, 70.

14. Lao, X. Q., et al., 2019. Long-term exposure to ambient fine particulate matter (PM(2.5)) and incident type 2 diabetes: a longitudinal cohort study. Diabetologia. 62, 759-769.

15. Lau, L. H., et al., 2019. Prevalence of diabetes and its effects on stroke outcomes: A meta-analysis and literature review. J Diabetes Investig. 10, 780-792.

16. Li, H., et al., 2018. Effects of ambient carbon monoxide on daily hospitalizations for cardiovascular disease: a time-stratified case-crossover study of 460,938 cases in Beijing, China from 2013 to 2017. Environ Health. 17, 82.

17. Li, J., et al., 2019. Association between ambient particulate matter air pollution and ST-elevation myocardial infarction: A case-crossover study in a Chinese city. Chemosphere. 219, 724-729.

18. Liang, F., et al., 2019. Long-term exposure to ambient fine particulate matter and incidence of diabetes in China: A cohort study. Environ Int. 126, 568-575.

19. Lin, H., et al., 2016. Mortality burden of ambient fine particulate air pollution in six Chinese cities: Results from the Pearl River Delta study. Environ Int. 96, 91-97.

20. Liu, H., et al., 2017a. Association between ambient air pollution and hospitalization for ischemic and hemorrhagic stroke in China: A multicity case-crossover study. Environ Pollut. 230, 234-241. 
21. Liu, H., et al., 2017b. Ambient Particulate Matter Concentrations and Hospitalization for Stroke in 26 Chinese Cities: A Case-Crossover Study. Stroke. 48, 2052-2059.

22. Lucht, S., et al., 2020. All-source and source-specific air pollution and 10-year diabetes Incidence: Total effect and mediation analyses in the Heinz Nixdorf recall study. Environ Int. 136, 105493.

23. Maji, K. J., et al., 2017. Burden of disease attributed to ambient PM2.5 and PM10 exposure in 190 cities in China. Environ Sci Pollut Res Int. 24, 11559-11572.

24. Maji, K. J., et al., 2018. Estimating premature mortality attributable to PM2.5 exposure and benefit of air pollution control policies in China for 2020. Sci Total Environ. 612, 683-693.

25. O'Donnell, M. J., et al., 2011. Fine particulate air pollution (PM2.5) and the risk of acute ischemic stroke. Epidemiology. 22, 422-31.

26. O'Neill, M. S., et al., 2005. Diabetes enhances vulnerability to particulate air pollution-associated impairment in vascular reactivity and endothelial function. Circulation. 111, 2913-20.

27. Paul, L. A., et al., 2020. The impact of air pollution on the incidence of diabetes and survival among prevalent diabetes cases. Environ Int. 134, 105333.

28. Rajagopalan, S., et al., 2018. Air Pollution and Cardiovascular Disease: JACC State-of-the-Art Review. J Am Coll Cardiol. 72, 2054-2070.

29. Saeedi, P., et al., 2019a. Global and regional diabetes prevalence estimates for 2019 and projections for 2030 and 2045: Results from the International Diabetes Federation Diabetes Atlas, 9(th) edition. Diabetes Res Clin Pract. 157, 107843.

30. Saeedi, P., et al., 2019b. Global and regional diabetes prevalence estimates for 2019 and projections for 2030 and 2045: results from the International Diabetes Federation Diabetes Atlas, $9^{\text {th }}$ edition. Diabetes Res Clin Pract. 157, 107843.

31. Shah, A. S., et al., 2015. Short term exposure to air pollution and stroke: systematic review and metaanalysis. BMJ. 350, h1295.

32. Song, J., et al., 2019. Acute effect of ambient air pollution on hospitalization in patients with hypertension: A time-series study in Shijiazhuang, China. Ecotoxicol Environ Saf. 170, $286-292$.

33. Song, J., et al., 2018. Acute effects of ambient particulate matter pollution on hospital admissions for mental and behavioral disorders: A time-series study in Shijiazhuang, China. Sci Total Environ. $636,205-211$.

34. Stafoggia, M., et al., 2017. Statistical Approaches to Address Multi-Pollutant Mixtures and Multiple Exposures: the State of the Science. Curr Environ Health Rep. 4, 481-490.

35. Tian, Y., et al., 2017. Fine Particulate Air Pollution and First Hospital Admissions for Ischemic Stroke in Beijing, China. Sci Rep. 7, 3897.

36. Villeneuve, P. J., et al., 2012. Short-term effects of ambient air pollution on stroke: who is most vulnerable? Sci Total Environ. 430, 193-201.

37. Wang, Q., et al., 2017a. Hypertension modifies the short-term effects of temperature on morbidity of hemorrhagic stroke. Sci Total Environ. 598, 198-203. 
38. Wang, W., et al., 2017b. Prevalence, Incidence, and Mortality of Stroke in China: Results from a Nationwide Population-Based Survey of 480687 Adults. Circulation. 135, 759-771.

39. Xia, X., et al., 2019. Prevalence and risk factors of stroke in the elderly in Northern China: data from the National Stroke Screening Survey. J Neurol. 266, 1449-1458.

40. Xu, X., et al., 2018. Progression of diabetes, heart disease, and stroke multimorbidity in middle-aged women: A 20-year cohort study. PLoS Med. 15, e1002516.

41. Xue, T., et al., 2019. A national case-crossover study on ambient ozone pollution and first-ever stroke among Chinese adults: Interpreting a weak association via differential susceptibility. Sci Total Environ. 654, 135-143.

42. Yang, B. Y., et al., 2020. Ambient air pollution and diabetes: A systematic review and meta-analysis. Environ Res. 180, 108817.

43. Yang, B. Y., et al., 2018a. Global association between ambient air pollution and blood pressure: A systematic review and meta-analysis. Environ Pollut. 235, 576-588.

44. Yang, B. Y., et al., 2018b. Ambient air pollution in relation to diabetes and glucose-homoeostasis markers in China: a cross-sectional study with findings from the 33 Communities Chinese Health Study. Lancet Planet Health. 2, e64-e73.

45. Yang, Y., et al., 2018c. Ambient fine particulate pollution associated with diabetes mellitus among the elderly aged 50 years and older in China. Environ Pollut. 243, 815-823.

46. Yu, Y., et al., 2019. Association between short-term exposure to particulate matter air pollution and cause-specific mortality in Changzhou, China. Environ Res. 170, 7-15.

47. Zeng, W., et al., 2018. Ambient fine particulate pollution and daily morbidity of stroke in Chengdu, China. PLoS One. 13, e0206836.

48. Zhou, M., et al., 2019. Mortality, morbidity, and risk factors in China and its provinces, 1990-2017: a systematic analysis for the Global Burden of Disease Study 2017. Lancet. 394, 1145-1158.

\section{Figures}


(a) Air pollutants

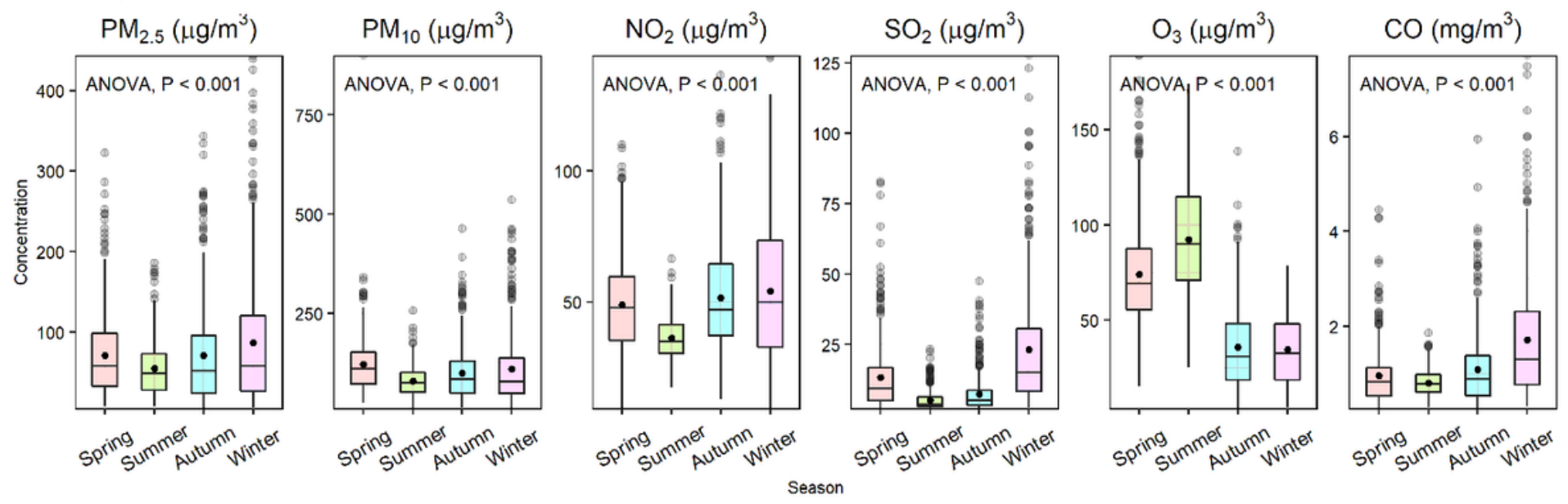

(b)Meteorological conditions

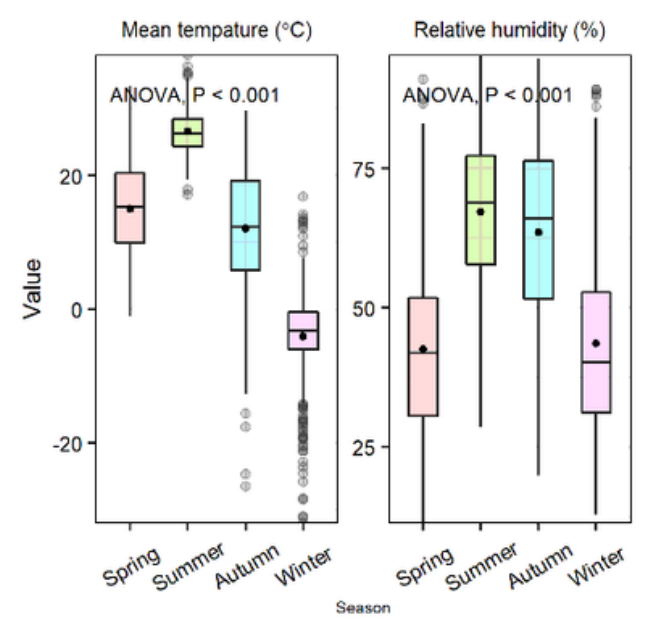

(c) Number of daily admissions for stroke

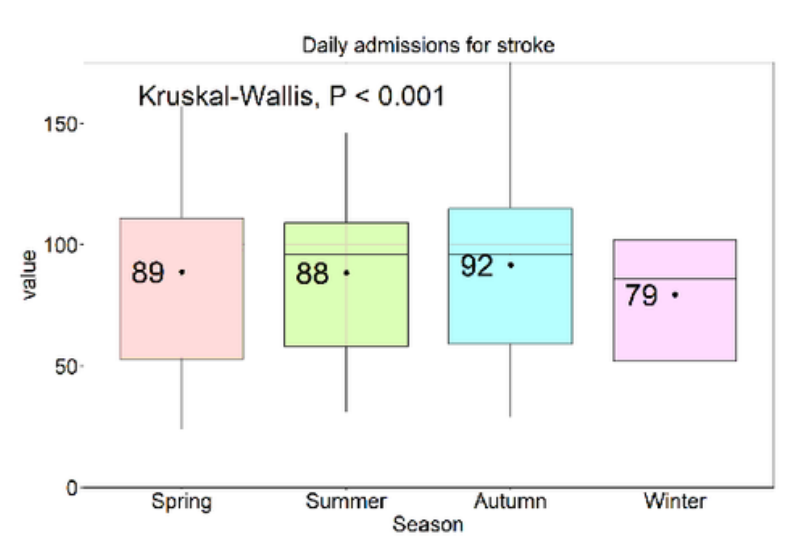

\section{Figure 1}

Boxplots of air pollutants, meteorological factors, and the daily counts of admissions for stroke during 2014 and 2018. (a) The seasonal pattern of air pollutants; (b) The seasonal pattern of meteorological conditions; (c) The seasonal pattern of daily admissions for stroke. Analysis of variance or Kruskal-Wallis tests were applied to examine the difference across four seasons. PM2.5: particles with an aerodynamic diameter $\leq 2.5 \mu \mathrm{m}$; PM10: particles with an aerodynamic diameter $\leq 10 \mu \mathrm{m} ; \mathrm{NO} 2$ : nitrogen dioxide; SO2: sulfur dioxide; O3: ozone; CO: carbon monoxide. 


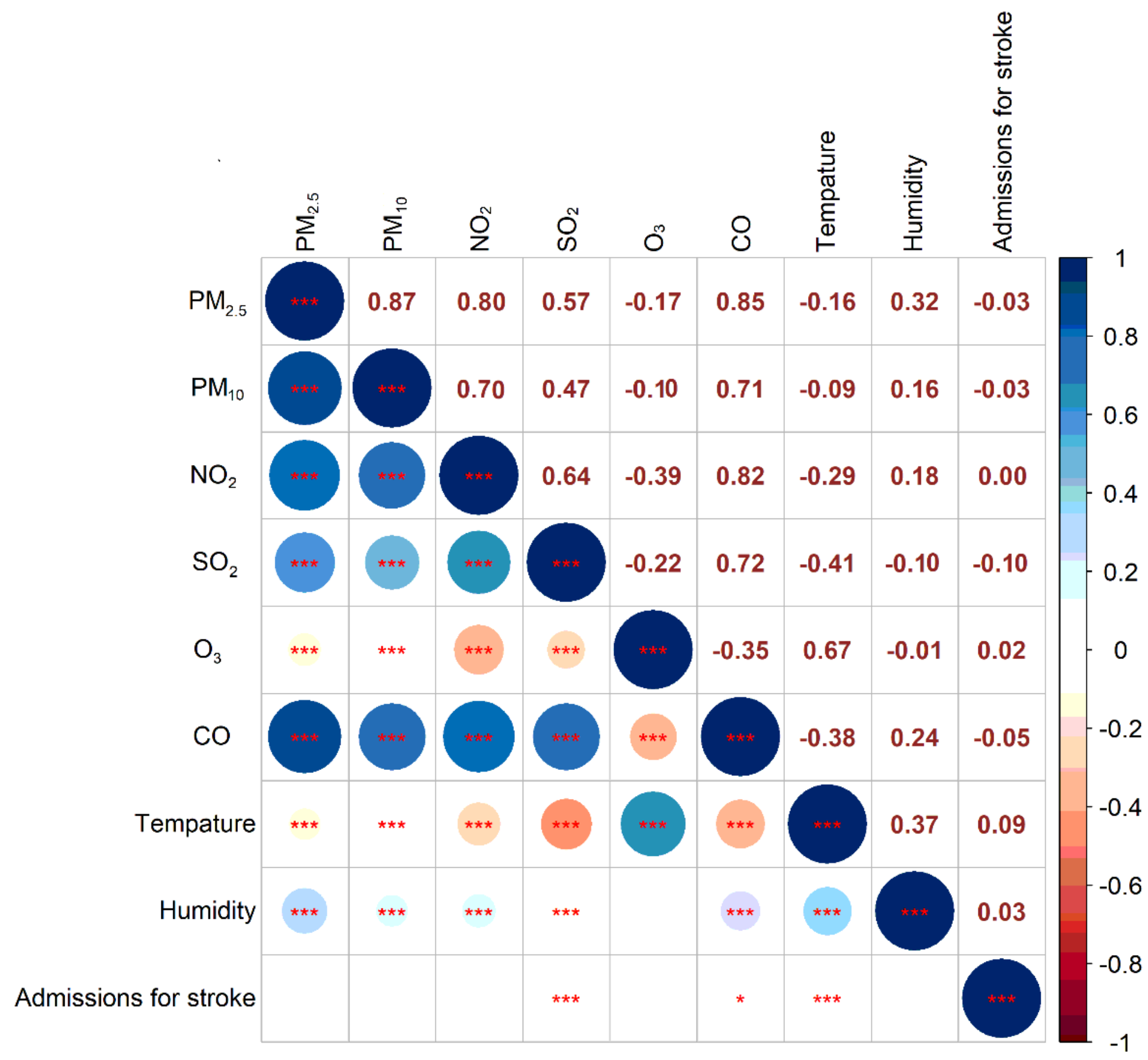

Figure 2

Pearson correlation coefficients between air pollution concentrations and weather factors and daily admissions for stroke among T2D patients in Beijing, 2014-2018 ( $n=1799)$. NO2: nitrogen dioxide; SO2: sulfur dioxide; 03: ozone; CO: carbon monoxide; PM2.5: particles with an aerodynamic diameter $\leq 2.5$ $\mu \mathrm{m}$; PM10: particles with an aerodynamic diameter $\leq 10 \mu \mathrm{m}$. * indicates $0.01<\mathrm{P} \leq 0.05$; $* \star$ indicates $0.001<P \leq 0.01 ; \star \star \star$ indicates $\mathrm{P} \leq 0.001$. 
(a) Overall stroke

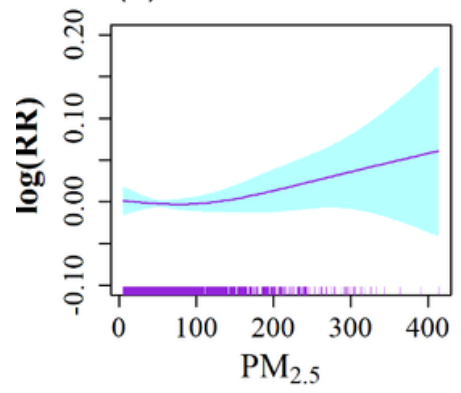

(e) Women

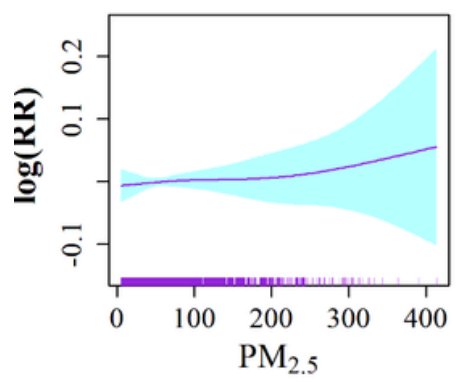

(b) Hemorrhagic stroke

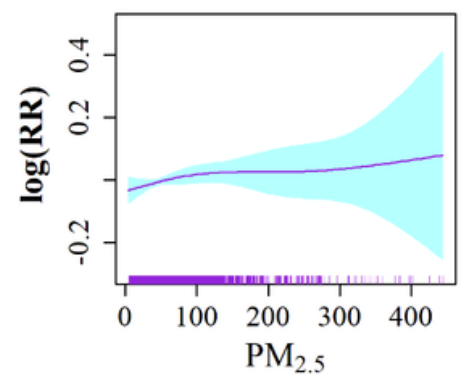

(f) Age 18-64 years

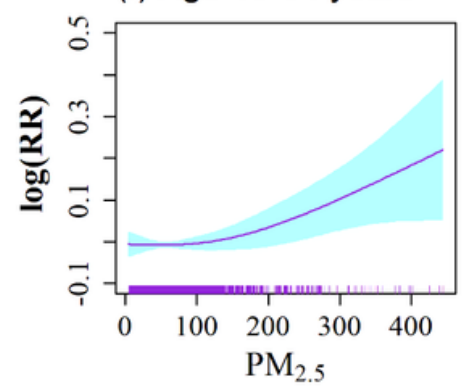

(c) Ischemic stroke

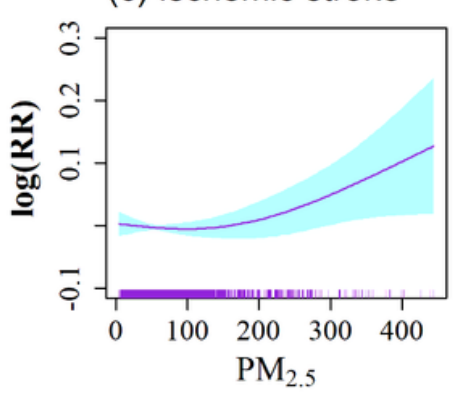

(g) Age $>=65$ years

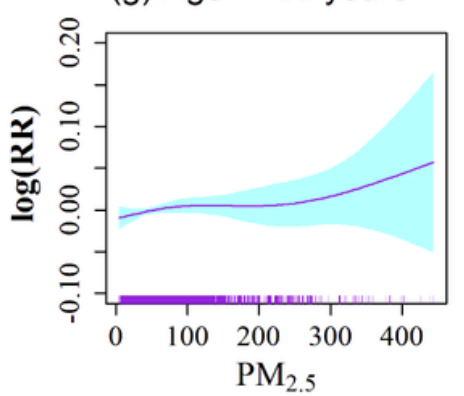

Figure 3

Dose-response relationship between PM2.5 (degrees of freedom = 3) and stroke admissions in Beijing, 2014-2018 using multi-pollutant model, after adjusted for temperature, relative humidity, day of week and public holidays.

(a) Overall stroke

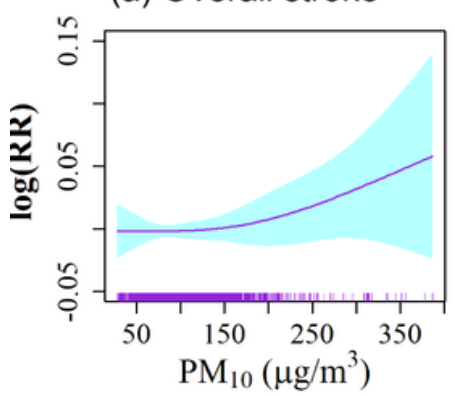

(e) Women

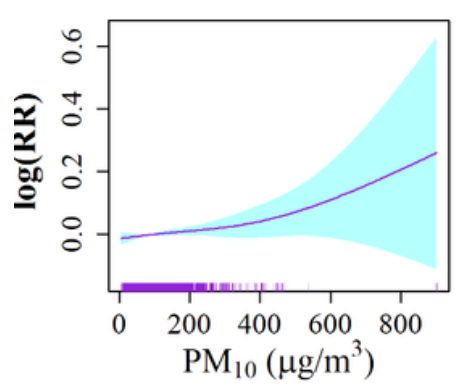

(b) Hemorrhagic stroke

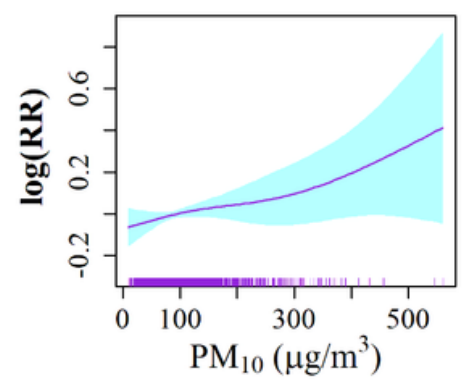

(f) Age $<65$ years

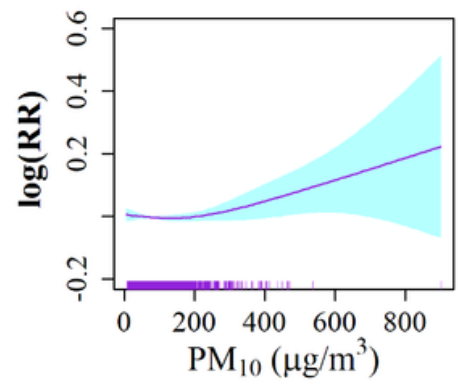

(c) Ischemic stroke

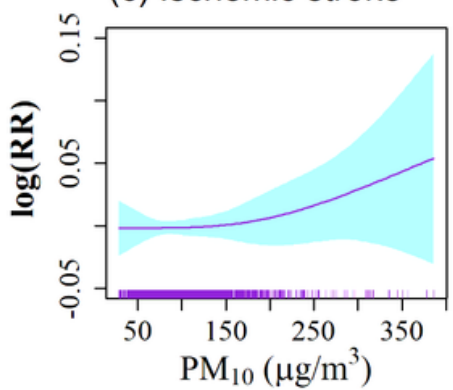

(g) Age $>=65$ years

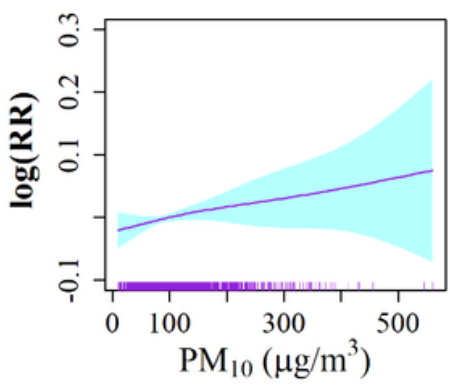

(d) Men

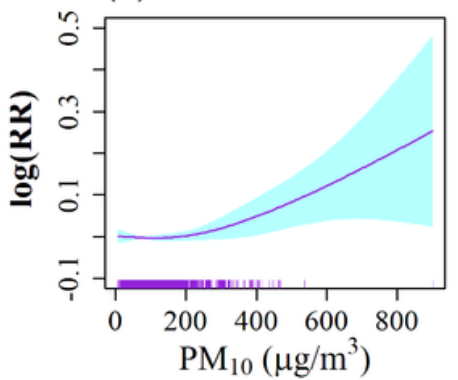

Figure 4 
Dose-response relationship between PM10 (degrees of freedom = 3) and stroke admissions in Beijing, 2014-2018 using multi-pollutant model, after adjusted for temperature, relative humidity, day of week and public holidays.

\section{Supplementary Files}

This is a list of supplementary files associated with this preprint. Click to download.

- 4Supplementalfile.docx 